\title{
DETERMINAÇÃo SIMULTÂNEA DE METAIS EM GASOLINA, ATRAVÉS DE MÉTODO ELETROANALÍTICO ALTERNATIVO, COM O USO DE CALIBRAÇÃO MULTIVARIADA / REDES NEURAIS*
}

\author{
SIMULTANEOUS DETERMINATION OF METALS IN GASOLINE THROUGH \\ ALTERNATIVE ELECTROANALYTICAL METHOD, WITH THE USE OF MULTIVARIATE \\ CALIBRATION / NEURAL NETWORKS
}

\author{
DETERMINACIÓN SIMULTÁNEA DE METALES EN GASOLINA, ATRAVES DE UN MÉTODO \\ ELECTROANALÍTICO ALTERNATIVO, USANDO CALIBRACIÓN \\ MULTIVARIADA / REDES NEURALES
}

Elmo de Sena Ferreira Júnior Joseany de Moraes S. Almeida Delano Brandes Marques Edmar Pereira Marques Aldaléa Lopes B. Marques

\begin{abstract}
Resumo: Devido a complexidade de amostras como a gasolina, a utilização de microemulsões, bem como de estratégias de calibração multivariada tem sido cada vez maior. Este trabalho apresenta o uso do programa STATISTICA 10.0 na aplicação de redes neurais artificiais (RNA) para prever a concentração dos íons metálicos $\mathrm{Pb}^{2+}$ e $\mathrm{Cd}^{2+}$ em amostras microemulsionadas de gasolina. Foi necessário descartar alguns dados experimentais de $\mathrm{Cd}^{2+}$, devido a baixa reprodutibilidade na determinação simultânea dos dois ions. Os dados foram complementados por interpolação a partir de medidas individuais de $\mathrm{Cd}^{2+}$, nas mesmas condições e no intervalo de $2 \times 10^{-8} \mathrm{~mol} \mathrm{~L}^{-1}$ a $6 \times 10^{-8} \mathrm{~mol} \mathrm{L^{-1 }}$. Através da otimização multivariada, os seguintes parâmetros ideais permitiram a obtenção de resposta simultânea dos metais pela técnica de voltametria de redissolução anódica em DP modo (DPASV): $\mathrm{E}_{\mathrm{dep}}=-1200 \mathrm{mV} ; \mathrm{t}_{\mathrm{dep}}=240 \mathrm{~s} ;$ Amplitude $=50 \mathrm{mV}$. Para o estudo de predição no presente trabalho, o tipo de RNA usado foi o MLP (Multi Layer Perceptron). Os valores das concentrações $\left(10^{-8} \mathrm{~mol} \mathrm{~L}^{-1}\right.$ a $10^{-7} \mathrm{~mol} \mathrm{~L}^{-1}$ ) e as respectivas correntes, determinados, experimentalmente, em condições otimizadas, e obtidos a partir de padrões dos dois ions, suplementaram, por interpolação dos dados, e foram usados como dados de entrada da rede, para ambos os analitos. As saídas dos modelos foram as concentrações dos analitos em amostras reais de gasolina. Estas amostras reais apresentaram arranjos do tipo MLP 1-4-1 para $\mathrm{Pb}^{2+}$, e MLP 1-2-1 para o $\mathrm{Cd}^{2+}$, sendo estes capazes de estimar a concentração na determinação simultânea dos dois metais na amostra de gasolina. Os valores simulados obtidos com a rede neural corresponderam estreitamente aos resultados experimentais esperados. Os valores médios de correlações foram considerados satisfatórios na previsão das concentrações de $C^{2+}\left(R_{2}=0,998\right)$ e $P b^{2+}\left(R_{2}\right.$ $=0,995)$. O eletrodo de filme de bismuto foi usado devido a sua baixa toxicidade e boa sensibilidade. Do ponto de vista analítico, o procedimento foi aplicado com sucesso em amostras reais de gasolina, no nível de $10^{-9}$ e $10^{-8} \mathrm{~mol} \mathrm{~L}^{-1}$ para os ions $\mathrm{Pb}^{2+}$ e $\mathrm{Cd}^{+}$, respectivamente.
\end{abstract}

Palavras-chave: Redes neurais artificiais. Planejamento experimental. Voltametria de redissolução anódica. Cobre. Chumbo. Gasolina.

\begin{abstract}
Due to the complexity of samples such as gasoline, the use of microemulsions, as well as the multivariate calibration strategies have been used increasingly. This work presents the use of STATISTICA Software 10.0 in the application of artificial neural networks (ANN) to predict the concentration of metal ions $\mathrm{Pb}^{2+}$ and $\mathrm{Cd}^{2+}$ in microemulsified samples of gasoline. It was necessary to dismiss some experimental data of $\mathrm{Cd}^{2+}$, due to low reproducibility in the simultaneous determination. The data were supplemented by interpolation from individual measures of $\mathrm{Cd}^{2+}$ in the same conditions and in the range of $2 \times 10^{-8} \mathrm{~mol}$ $\mathrm{L}^{-1}$ to $6 \times 10^{-8} \mathrm{~mol} \mathrm{~L}^{-1}$. Through multivariate optimization, the following optimal parameters allowed to obtain simultaneous response of metals by anodic stripping voltammetry technique in DP mode: $E_{\text {dep }}=-1200$ $\mathrm{mV} ; \mathrm{t}_{\mathrm{dep}}=240 \mathrm{~s} ; \mathrm{Amp}=50 \mathrm{mV}$. For the prediction study in the present work, the type of ANN used was the MLP (Multi Layer Perceptron). Values of concentrations $\left(10^{-8} \mathrm{~mol} \mathrm{~L}^{-1}\right.$ to $\left.10^{-7} \mathrm{~mol} \mathrm{~L}^{-1}\right)$ and respective currents, determined experimentally in optimized conditions, and obtained from standard of the two metals, supplemented the data by interpolation, and were used as input data in the network, for both analytes. The outputs of the models were the concentrations of the analytes in real samples of gasoline. This real sample, presented a MLP arrangement of the type 1-4-1 for $\mathrm{Pb}^{2+}$, and 1-2-1 for $\mathrm{Cd}^{2+}$, being them able to estimate the concentrations of simultaneous determination of the two metals in gasoline sample. The simulated values obtained with the neural network, corresponded closely to the experimental results expected. Mean values of correlations, considered very satisfactory, were obtained in the sample gasoline, in the prediction of the concentrations of $\mathrm{Cd}^{2+}\left(R_{2}=0.998\right)$ and $\mathrm{Pb}^{2+}\left(R_{2}=0.995\right)$. The bismuth film electrode has been used due to its low toxicity and good sensitivity. From the analytical point of view, the procedure was applied
\end{abstract}

Trabalho premiado durante o XXIV Encontro do SEMIC, realizado na UFMA entre os dias 05 a 08 de novembro de 2012.

*Artigo recebido em dezembro 2012

Aprovado em fevereiro 2013 
successfully to the real samples of gasoline at the level of $10^{-9}$ and $10^{-8} \mathrm{~mol} \mathrm{~L}^{-1}$ for the ions $\mathrm{Pb}^{2+}$ and $\mathrm{Cd}^{2+}$, respectively.

Keywords: Artificial neural networks. Experimental design. Anodic stripping voltammetry. Copper. Lead. Gasoline.

Resumen: Debido a la complexidad de muestras, tales como la gasolina, la utilización de microemulsiones, asi como también de estrategias de calibración multivariada, están siendo cada vez mas comunes. Este trabajo presenta el empleo del programa STATISTICA 10.0 en la aplicación de redes neurales artificiales (RNA), para visualizar anticipadamente la concentración de los iones metálicos $\mathrm{Pb}^{2+}$ y $\mathrm{Cd}^{2+}$ en muestras microemulsionadas de gasolina. Fue necesario descartar algunos datos experimentales de Cd2+ debido a la baja reproductibilidad en la determinación simultánes de los dos iones. Los datos fueron complementados por interpolación a partir de mediciones individuales de $\mathrm{Cd}^{2+}$, en las mismas condiciones, y en el intervalo de $2 \times 10^{-8} \mathrm{~mol} \mathrm{~L}^{-1}$ a $6 \times 10^{-8} \mathrm{~mol}^{2}$ $\mathrm{L}^{-1}$. Atraves de la optimización multivariada, los siguientes parámetros ideales permitieron obtener la respuesta simultánea de los metales por la técnica de voltametría de redisolución anódica en DP modo (DPASV): $\mathrm{E}_{\mathrm{dep}}=$ $-1200 \mathrm{mV} ; \mathrm{t}_{\mathrm{dep}}=240 \mathrm{~s} ;$ Amplitude $=50 \mathrm{mV}$. Para el estudio de visualización anticipada en este trabjo, el tipo

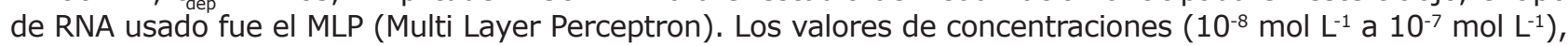
y las respectivas corrientes, determinados experimentalmente en condiciones optimizadas, y obtenidos a partir de muestras standard de los dos iones, suplementaron por interpolación de los datos, y fueron usados como informaciones de entrada de la red, para ambos analitos. Las salidas de los modelos fueron las concentraciones de los analitos en muestras reales de gasolina. Estas muestras reales presentaron organizaciones del tipo MLP 1-4-1 para $\mathrm{Pb}^{2+}$, e MLP 1-2-1 para o $\mathrm{Cd}^{2+}$, lo que permite estimar la concentración en la determinación simultánea de los dos metales en la muestra de gasolina. Los valores simulados obtenidos con la red neural corriespondieron muy proximamente a los resultados experimentales esperados. Los valores medios de las correlaciones, considerados muy satisfactorios, fueron obtenidos en el gas de la muestra, la previsión de las

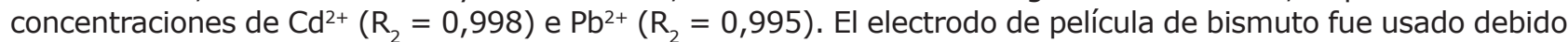
a sua baja toxicidad y buena sensibilidad. Del punto de vista analítico, el procedimiento fue aplicado con éxito en muestras reales de gasolina, en el nivel de $10^{-9}$ y $10^{-8} \mathrm{~mol} \mathrm{~L}^{-1}$ para los iones $\mathrm{Pb}^{2+}$ e $\mathrm{Cd}^{2+}$, respectivamente.

Palabras clave: Redes neurales artificiales. Planeamento experimental. Voltametria de redisolução anódica. Cobre. Plomo. Gasolina.

\section{INTRODUÇÃo}

A gasolina é um combustível automotivo, derivado do petróleo, constituído de uma mistura complexa de hidrocarbonetos (normalmente 4 a 12 átomos de carbono) e em menor quantidade por produtos oxigenados, compostos de enxofre, de nitrogênio e compostos metálicos em baixas concentrações, que conferem certo grau de instabilidade ao produto. (BORSATO et al., 2012). A Resolução ANP No 57, de 20.10.2011 (AGÊNCIA NACIONAL DO PETRÓLEO, GÁS NATURAL E BIOCOMBUSTÍVEIS, 2011), estabelece as especificações para a comercialização de gasolinas automotivas em todo o território nacional e regulamenta as especificações das gasolinas de uso automotivo e as obrigações quanto ao controle da qualidade a serem atendidas pelos diversos agentes econômicos que comercializam o produto em todo o território nacional.

Muitos elementos metálicos são constituintes naturais dos derivados de petróleo, como a gasolina, mas também podem ser incorporados durante as etapas de processamento do produto, através do contato com os equipamentos usados no processo de refino ou destilação, estocagem e transporte. (SAINT' PIERRE et al.,2004; AUCÉLIO; CURTIUS, 2002). A presença de metais é indesejável, mesmo em baixas concentrações (CHAVES et al., 2008; AUCÉLIO et al., 2004; MARTINIANO et al., 2012) e a determinação destes elementos, mesmo em quantidades a nível de traços em derivados de petróleo é importante devido à sua influência na estabilidade e no desempenho do combustível. Além disso, é importante a avaliação das quantidades desses elementos que são emitidos na atmosfera quando o combustível é queimado. (MARQUES et al., 2012; REYES; CAMPOS, 2005; SANZ-MEDEL et al., 1999)

O chumbo e o cádmio são grandes contaminantes ambientais. O chumbo começou a ser misturado à gasolina a partir de 1922 , com o objetivo de melhorar o comportamento dos motores, conferindo-Ihes basicamente aumento de octanagem para suprir as necessidades dos motores do ciclo OTTO. Já no início desta aplicação, notou-se o poder de contaminação que o chumbo poderia trazer junto aos canalizadores de gases que tiveram que ser removidos aumentando assim os níveis de contaminação atmosférica.

A partir de 1970 começaram algumas discussões tratando da remoção do chumbo da gasolina. O objetivo era reduzir sua emissão e eliminar as contaminações de ar, solo e água que ocorriam devido a essa adição. (LANDRIGAN, 2002).

Já o cádmio destaca-se pela sua alta toxicidade e uso tecnológico crescente, podendo ser absorvido por via respiratória e intestinal. Este metal apresenta também dificuldades de eliminação sendo excretado pelos rins. O alto grau de toxidade pode causar náuseas, vômitos, diarreia, anemia, enfisema pulmonar e câncer. (FERREIRA et al., 2005). 
A voltametria compreende um conjunto de métodos eletroanalíticos, nos quais as informações qualitativas e quantitativas de uma espécie são obtidas a partir de medidas de corrente em função do potencial aplicado. A medida experimental é feita durante a eletrólise da espécie de interesse em uma célula eletroquímica, com três eletrodos (trabalho, auxiliar e referência). (BARD; FAULKNER, 2001).

As técnicas eletroanalíticas são capazes de fornecer limites de detecção excepcionalmente baixos e uma abundância de informações como: estequiometria, velocidade de transferência de carga interfacial e de massa, entre outras, que caracterizam e descrevem eletroquimicamente determinados sistemas. (CHRISTIAN, 1986).

\section{Quimiometria}

O uso de ferramentas estatísticas, matemáticas e gráficas para resolver sistemas químicos deu origem a uma área da química conhecida como quimiometria. A quimiometria pode ser útil tanto no planejamento e otimização de experimentos quanto na análise de resultados para obtenção da maior quantidade de dados possíveis. (FERREIRA et al., 2005).

\section{Planejamento fatorial experimental}

Dentre as várias alternativas existentes para planejamento experimental, destacam-se os sistemas de planejamento fatorial, os quais permitem avaliar simultaneamente o efeito de um grande número de variáveis, a partir de um número reduzido de ensaios experimentais. A teoria em que esses sistemas se baseiam é bastante simples e está disponível na literatura especializada. (BARROS NETO; SCARMINIO; BRUNS, 2001). O planejamento fatorial experimental é uma ferramenta estatística que, por sua simplicidade, vem sendo cada vez mais utilizada pelos químicos analíticos, pois permite avaliar as condições ideais de trabalho e a otimização de metodologias. (COSTA et al., 2006). Um planejamento adequado permite também a redução da variabilidade de resultados, a redução do tempo de análise e dos custos envolvidos. (BUTTON, 2005).

Planejamento fatorial é um tipo de planejamento experimental que é de grande utilização em investigações preliminares quando se deseja saber se determinados fatores têm ou não influência sobre a resposta desejada, e não se está preocupado com uma descrição muito rigorosa dessa influência. (BARROS NETO; SCARMINIO; BRUNS, 1996).

Para executar um planejamento fatorial experimental é preciso determinar quais os fatores (variáveis independentes) e qual a resposta de interesse (variável dependente) para o sistema que se deseja estudar. Também é preciso definir que objetivo se quer alcançar com os experimentos, porque isto definirá que tipo de planejamento deverá ser utilizado. Devido a sua simplicidade e baixo custo, o planejamento fatorial de dois níveis, completo ou fracionário (COSTA et al., 2006) é muito utilizado em estudos preliminares ou como passo inicial para uma otimização. Neste tipo de planejamento, cada fator é estudado em dois níveis (máximo e mínimo) e, portanto, supõese uma linearidade nos efeitos dos fatores.

A metodologia de superfície de resposta (RSM, Response Surface Methodology) é uma técnica de otimização baseada no emprego de planejamentos fatoriais (BARROS NETO; SCARMINIO; BRUNS, 1996) e tem sido usada com grande sucesso na modelagem de diversos processos. Uma superfície de resposta é um gráfico que mostra o comportamento da resposta como função de dois ou mais fatores. A superfície de resposta oferece uma maneira conveniente de visualizar como os fatores afetam as medidas do sistema. (CALADO; MONTGOMERY, 2003; MONTGOMERY, 1991). Para a obtenção de uma superfície de resposta não linear, e obtenção de um ponto ótimo, é necessário avaliação de um considerável número de pontos. (BARROS NETO; SCARMINIO; BRUNS, 1996; CALADO; MONTGOMERY, 2003).

A técnica do planejamento fatorial, junto com a metodologia da superfície de resposta tem se mostrado eficiente para quantificar o impacto das incertezas dos reservatórios na previsão da produção. Estes procedimentos têm sido aplicados com sucesso, na análise de metais em combustíveis, tanto usando técnicas espectroscópicas (TRINDADE et al., 2006) como técnicas eletroanalíticas (TRINDADE et al., 2012). O método do planejamento fatorial possibilita estimar o impacto das incertezas e é útil para a melhor compreensão do sistema, permitindo a análise individual do efeito de cada variável na resposta desejada. (RODRIGUES; IEMMA, 2005).

\section{Redes neurais artificiais}

As redes neurais artificiais (RNA's) tiveram inspiração a partir dos sistemas biológicos. Um neurônio biológico pode ser representado por suas sinapses, que são junções nas quais um sinal é passado de um neurônio para outro, e pela força sináptica, que é a magnitude da influência do sinal para o próximo neurônio. As ligações sinápticas entre dois neurônios biológicos são representadas pelas conexões entre dois neurônios artificiais nas RNA's, e a força sináptica é representada por um peso associado a cada conexão. Ambos os sistemas, biológicos ou artificiais, usam um grande número de processadores simples com alto grau de conectividade e processam a informação através de eventos relativamente discretos. Utilizam também uma forma distribuída de representação ou memória. São sistemas adaptivos que aprendem pelo ajuste da força e número de conexões entre neurônios (WYTHOFF, 1993). As RNA's são estruturas matemáticas utilizadas em processamento de dados complexos, as quais podem ajustar ou modelar o comportamento não linear de muitos processos químicos.

Duas importantes características das RNA são a sua habilidade de fornecer respostas 
rápidas a um problema e a capacidade de generalizar suas respostas, fornecendo resultados confiáveis para amostras desconhecidas (SERRA, et al., 2003).

Existem muitos tipos de RNA's que foram desenvolvidas até a presente data e a escolha do tipo depende basicamente do problema a ser resolvido. A arquitetura ou topologia de uma RNA compreende o número de neurônios em cada camada, tipo de conexão entre os neurônios e o número de camadas existentes. (HAYKIN, 1999).

No presente trabalho, um planejamento experimental, associado às redes neurais artificiais, é aplicado a um procedimento eletroanalítico, com o objetivo de viabilizar as melhores condições e resultados, na determinação simultânea de metais em amostras microemulsionadas de gasolina.

\section{PARTE EXPERIMENTAL}

\section{Instrumentação}

As medidas voltamétricas foram realizadas em um sistema eletroanalítico AUTOLAB PGSTAT 12/30/320 acoplado a um computador Celeron Intel.

Os eletrodos de filme de bismuto (BiFE) e filme de mercúrio (MFE) foram preparados por eletrodeposição eletroquímica, mediante a aplicação de um adequado potencial de redução dos íons $\mathrm{Bi}^{3+}$ e $\mathrm{Hg}^{2+}$, em um adequado eletrólito suporte e durante um determinado tempo de deposição.

\section{Soluções e reagentes}

Todos os reagentes químicos empregados foram de grau analítico. A água usada foi deionizada em um Purificador Nanopure modelo Barnstead acoplado a um destilador de Água Quimis. O reagente usado foi o ácido nítrico supra-puro (MERCK, Darmstadt, Alemanha).

A solução de $\mathrm{Pb}^{2+}$ e $\mathrm{Cd}^{2+}$ foi preparada a partir da diluição dos padrões concentrados de cada metal, de $1000 \mathrm{mg} \mathrm{L}^{-1}$, de procedência da MERCK.

Para o preparo do filme BiFE foi usada a solução padrão de nitrato de bismuto $\mathrm{Bi}\left(\mathrm{NO}_{3}\right)_{3}$ ' com concentração de $1000 \mathrm{mg} \mathrm{L}^{-1}$. O filme de $\mathrm{Hg}$ foi preparado a partir de uma solução de $\mathrm{Hg}\left(\mathrm{NO}_{3}\right)_{2} \mathrm{com}$ concentração de $1000 \mathrm{mg} \mathrm{L}^{-1}$.

A solução de tampão acetato foi preparada a partir das soluções de ácido acético e acetato de sódio, ambas na concentração de $0,1 \mathrm{~mol} \mathrm{~L}^{-1}$, para um volume de $1 \mathrm{~L}$.

A desaeração das soluções realizadas durante as medidas voltamétricas foi realizada empregando-se o gás nitrogênio seco de procedência WHITE MARTINS, com pureza 4.6 FID, durante 5 minutos.

\section{Preparo das amostras}

Para o preparo das microemulsões foi feita uma mistura de três componentes (Gasolina: propan-1-ol: ácido nítrico). O preparo das microemulsões consistiu, inicialmente, da mistura de $1,0 \mathrm{~mL}$ de $\mathrm{HNO}_{3} 1 \times 10^{-7} \mathrm{~mol} \mathrm{~L}^{-1} \mathrm{com}$ $5,0 \mathrm{~mL}$ de gasolina, sob agitação de 5 minutos. Logo depois foram adicionados $12 \mathrm{~mL}$ de propano-1-ol sob uma leve agitação para a homogeneização. Essas proporções foram obtidas por meio de testes de proporcionalidade, baseando-se no procedimento apresentado pela literatura. (CARDOSO et al., 2007; CARDOSO et al., 2010). A formação da microemulsão foi observada através da transparência visual.

\section{RESULTADOS E DISCUSSÃO}

Otimização do sistema eletroanalítico

A medida eletroanalítica é influenciada por diferentes parâmetros operacionais, como, por exemplo, os parâmetros selecionados para estudo neste trabalho. Desta forma a construção de uma matriz de planejamento como a mostrada na tabela 1 é de fundamental importância, onde se observa as combinações dos níveis das variáveis. Seguindo-se a ordem aleatória dos experimentos obteve-se as respostas analíticas (corrente do pico), para cada metal, como pode ser observado na tabela 2 .

Tabela 1 - Matriz fatorial 23 completo para otimização do sistema eletroanalítico

\begin{tabular}{c|c|c}
\hline \multirow{2}{*}{ ORDEM } & \multicolumn{2}{|c}{$\begin{array}{c}\text { VARIÁVEIS DEPENDENTES } \\
\text { (CORRENTE DO PICO/A) }\end{array}$} \\
\cline { 2 - 3 } & Cd $^{2+}$ & $\mathrm{Pb}^{2+}$ \\
\hline 1 & $1,68 \mathrm{E}-07$ & $5,09 \mathrm{E}-06$ \\
\hline 2 & $2,23 \mathrm{E}-07$ & $5,06 \mathrm{E}-06$ \\
\hline 3 & $1,14 \mathrm{E}-05$ & $5,02 \mathrm{E}-06$ \\
\hline 4 & $1,59 \mathrm{E}-05$ & $3,31 \mathrm{E}-06$ \\
\hline 5 & $5,47 \mathrm{E}-07$ & $1,29 \mathrm{E}-05$ \\
\hline 6 & $6,02 \mathrm{E}-07$ & $1,31 \mathrm{E}-05$ \\
\hline 7 & $3,50 \mathrm{E}-05$ & $1,35 \mathrm{E}-05$ \\
\hline 8 & $3,89 \mathrm{E}-05$ & $1,37 \mathrm{E}-05$ \\
\hline
\end{tabular}

Fonte: Elaborada pelos autores

Tabela 2 - Resultados dos experimentos da matriz do planejamento fatorial

\begin{tabular}{cccc}
\hline \multirow{2}{*}{ ORDEM } & \multicolumn{3}{c}{ VARIÁVEIS INDEPENDENTES } \\
\cline { 2 - 4 } & $\begin{array}{c}\text { Potencial de } \\
\text { deposição } \\
(\mathrm{mV})\end{array}$ & $\begin{array}{c}\text { Tempo de } \\
\text { deposição }(\mathrm{s})\end{array}$ & $\begin{array}{c}\text { Amplitude } \\
(\mathrm{mV})\end{array}$ \\
\cline { 1 - 3 } $\mathbf{1}$ & -1400 & 240 & 25 \\
\hline 2 & -1200 & 240 & 25 \\
\hline 3 & -1400 & 200 & 25 \\
\hline 4 & -1200 & 200 & 25 \\
\hline 5 & -1400 & 240 & 50 \\
\hline 6 & -1200 & 240 & 50 \\
\hline 7 & -1400 & 200 & 50 \\
8 & -1200 & 200 & 50 \\
\hline
\end{tabular}

Fonte: Elaborada pelos autores 
A análise de variância aplicada aos resultados da matriz de planejamento experimental (Tabela 2) é mostrada no gráfico de Pareto (Figura 1) e na figura 2 para cada metal. Os fatores menores que $95 \%$ de confiança não apresentam efeito significativo.

Para o $\mathrm{Cd}^{2+}$ usou-se a corrente de pico como resposta, e observou-se que somente o tempo de deposição apresentou um baixo valor significativo. Entretanto, o potencial de deposição foi a única variável que apresentou efeito significativo e negativo, ou seja, melhores valores de corrente do pico de $\mathrm{Cd}^{2+}$ podem ser obtidos no menor nível estabelecido no planejamento experimental. Também é notório que a interação entre o potencial de deposição (2) e a amplitude (3) tem um efeito significativo no experimento, justificando, desta forma, a utilização da otimização multivariada. No caso do íon $\mathrm{Pb}^{2+}$, a única variável de valor significativo foi à amplitude de pulso, com o seu melhor valor obtido no maior nível do planejamento experimental.

Figura 1 - Gráfico de Pareto para $\mathrm{Cd}^{2+}$

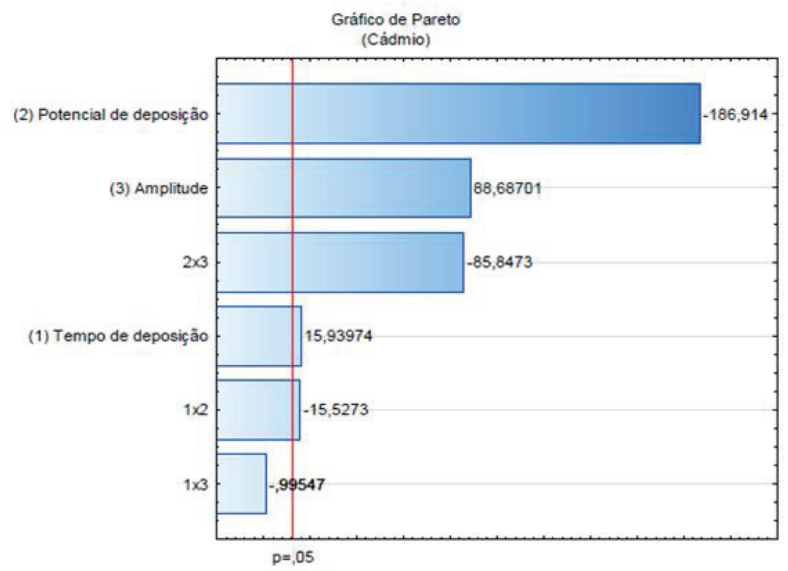

Fonte: Elaborada pelos autores

Figura 2 - Gráfico de Pareto para $\mathrm{Pb}^{2+}$

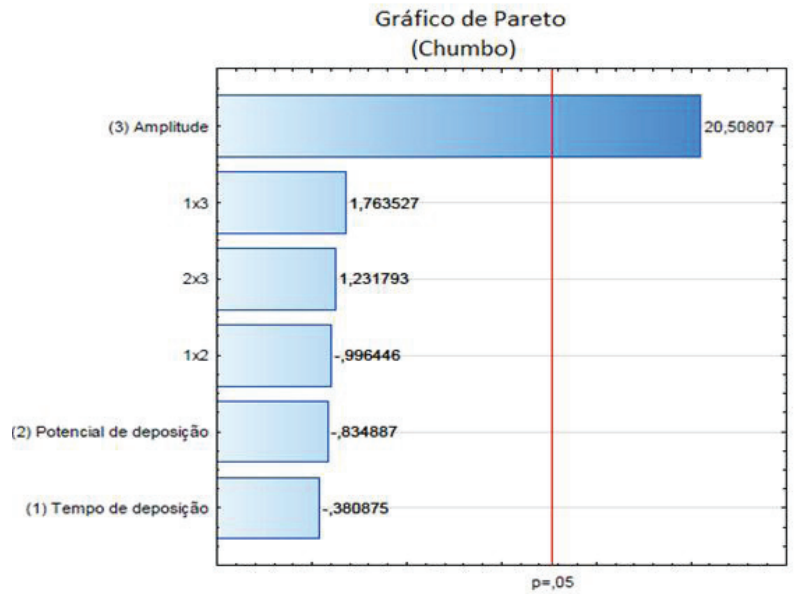

Fonte: Elaborada pelos autores

A partir dos dados obtidos pela curva de calibração (parâmetros de mérito apresenta- dos na tabela 2), para cada íon $\left(\mathrm{Pb}^{2+}\right.$ e $\left.\mathrm{Cd}^{2+}\right)$ foi possível calcular a concentração de cada metal. O limite de detecção (LD) foi calculado como sendo três vezes o desvio padrão obtido de 5 leituras consecutivas do branco, dividido pela inclinação da curva de calibração (coeficiente angular) e o valor de $0,0981 \mathrm{mgL}^{-1}$ mostra que a técnica é bastante sensível para a quantificação de $\mathrm{Pb}^{2+}$, mesmo em pequenas concentrações, podendo-se determinar a concentração do analito com segurança.

A precisão é a expressão da concordância entre vários resultados analíticos obtidos para uma mesma amostra. Geralmente é medida através do desvio padrão ou desvio padrão relativo. Neste trabalho a precisão medida pelo desvio padrão relativo (RSD) de 5 medidas da amostra teve como valor obtido de $20,23 \%$, o que demonstra uma boa precisão e com uma baixa variação entre os valores encontrados para o método aplicado, pois este valor encontra-se dentro da faixa permitida para métodos de análise de traços ou impurezas.

A figura 3 ilustra graficamente as variações dos efeitos principais: amplitude, tempo e potencial de deposição em função de seus níveis. Os gráficos dos efeitos principais ajudam perceber melhor as variáveis que são significativas nas respostas analíticas e também o comportamento na faixa dos níveis estudados.

Figura 3 - Gráficos de efeitos principais das variáveis para cada metal em estudo
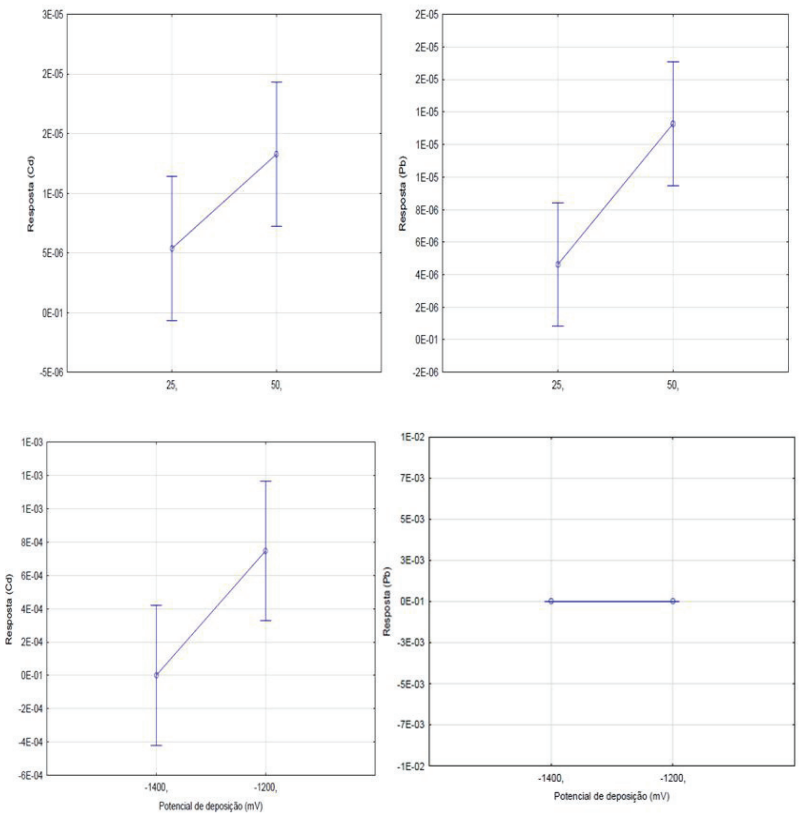

Fonte: Elaborada pelos autores

Também se observa na figura 3 para o metal cádmio que a amplitude de $50 \mathrm{mV}$ fornece um melhor resultado (nível menor estabelecido) e que esta variável apresenta um efeito significativo devido a grande inclinação da reta mostrando uma variação acentuada na resposta analítica. Interpretação semelhante pode ser utilizada para os outros fatores. 
Para o metal chumbo a faixa de potencial deposição não influencia significativamente em sua resposta analítica, visto que a reta possui inclinação nula.

Tabela 3 - Parâmetros otimizados para a determinação simultânea dos metais $\mathrm{Cd}$ e $\mathrm{Pb}$ em gasolina por voltametria de redissolução anódica.

\begin{tabular}{c|c}
\hline Parâmetros & Valores otimizados \\
\hline Potencial de deposição & $-1200 \mathrm{mV}$ \\
\hline Tempo de deposição & $240 \mathrm{~s}$ \\
\hline Amplitude & $50 \mathrm{mV}$ \\
\hline
\end{tabular}

Fonte: Elaborada pelos autores

A tabela 5 apresenta os parâmetros otimizados para o método proposto em estudo nas condições de trabalho em que foram executadas.

\section{Meio eletrolítico para determinação de metais}

Experimentos voltamétricos foram realizados usando-se $10 \mathrm{~mL}$ de um meio microemulsionado composto por gasolina, propanol e $\mathrm{HNO}_{3}(\mathrm{pH}=4,3)$. Neste meio, adicionaram-se alíquotas de soluções padrão dos metais. A figura 4 mostra a resposta voltamétrica para o $\mathrm{Cd}^{2+}$ e $\mathrm{Pb}^{2+}$.

Figura 4 - Voltamogramas de resposta simultânea de $\mathrm{Cd}^{2+}$ e $\mathrm{Pb}^{2+}$ em $10 \mathrm{~mL}$ de microemulsão $\left(\mathrm{HNO}_{3}\right.$ $0,7 \mathrm{~mol} \mathrm{~L}^{-1}$, gasolina e propanol; proporção de 15:25:60). Parâmetros: $\mathrm{pH}=4,3 ; \mathrm{E}_{\text {dep }}=-1200 \mathrm{mV}$; $\mathrm{t}_{\mathrm{dep}}=240 \mathrm{~s} ; \mathrm{Amp}=50 \mathrm{mV}$. $\left[\mathrm{Cd}^{2+}\right]$ : Sucessivas adiçỗes de solução padrão de $\mathrm{Cd}^{2+}$, de $2 \times 10^{-8} \mathrm{~mol} \mathrm{~L}^{-1}$ a $6 \times 10^{-8} \mathrm{~mol} \mathrm{~L}^{-1} ;\left[\mathrm{Pb}^{2+}\right]$ : Sucessivas adições de solução padrão de $\mathrm{Pb}^{2+}$, de $2 \times 10^{-8} \mathrm{~mol} \mathrm{~L}^{-1}$ a $6 \times 10^{-8} \mathrm{~mol} \mathrm{~L}^{-1}$

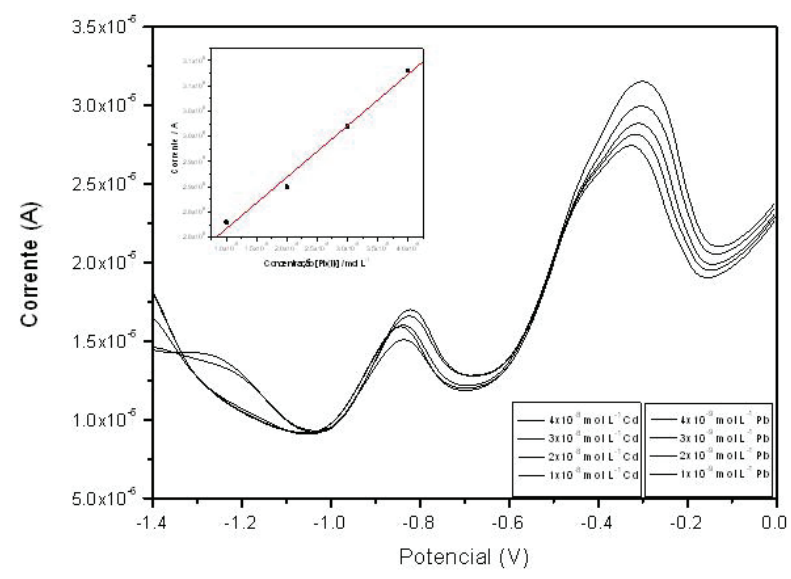

Fonte: Elaborada pelos autores

Conforme pode ser observado (Figura 4) a resposta voltamétrica para $\mathrm{Pb}^{2+}$ foi boa, em termos de resolução e sensibilidade, o que foi indicado pelo significativo aumento de corrente em função da adição de alíquotas sucessivas de $\mathrm{Pb}^{2+}$ na microemulsão estudada. O estudo foi realizado variando-se a concentração de $\mathrm{Pb}^{2+}$ entre $1 \times 10^{-9} \mathrm{~mol} \mathrm{~L}^{-1}$ a $4 \times 10^{-9} \mathrm{~mol} \mathrm{~L}^{-1}$, e a partir desses dados pôde-se construir uma curva analítica, que apresentou uma linearidade com coeficiente de correlação de 0.9834 e equação da reta $A=2,715 \mathrm{e}^{-5}+1020\left[\mathrm{~Pb}^{2+}\right]$.

No entanto, mesmo com o aumento da corrente em função de algumas das adições, a resposta do cádmio não teve uma boa resolução para determinação simultânea devido à complexidade da amostra e à interferência destes metais. Em contra partida, optou-se em obter reposta voltamétricas somente 0 $\mathrm{Cd}^{2+}$ com o objetivo de garantir a linearidade do sistema. A figura 5 mostra a resposta voltamétrica para o $\mathrm{Cd}^{2+}$.

Figura 5 - Voltamogramas de resposta de $\mathrm{Cd}^{2+}$ em $10 \mathrm{~mL}$ de microemulsão $\left(\mathrm{HNO}_{3}, 0,7 \mathrm{~mol} \mathrm{~L}^{-1}\right.$, gasolina e propanol; proporção de 15:25:60. Parâmetros: $\mathrm{pH}=4,3 ; \mathrm{E}_{\text {dep }}=-1200 \mathrm{mV} ; \mathrm{t}_{\text {dep }}=240 \mathrm{~s} ;$ Amp $=50$ mV. $\left[\mathrm{Cd}^{2+}\right]$ : Sucessivas adições de solução padrão de $\mathrm{Cd}^{2+}$, de $4 \times 10^{-8} \mathrm{~mol} \mathrm{~L}^{-1}$ a $1.8 \times 10^{-7} \mathrm{~mol} \mathrm{~L}^{-1}$

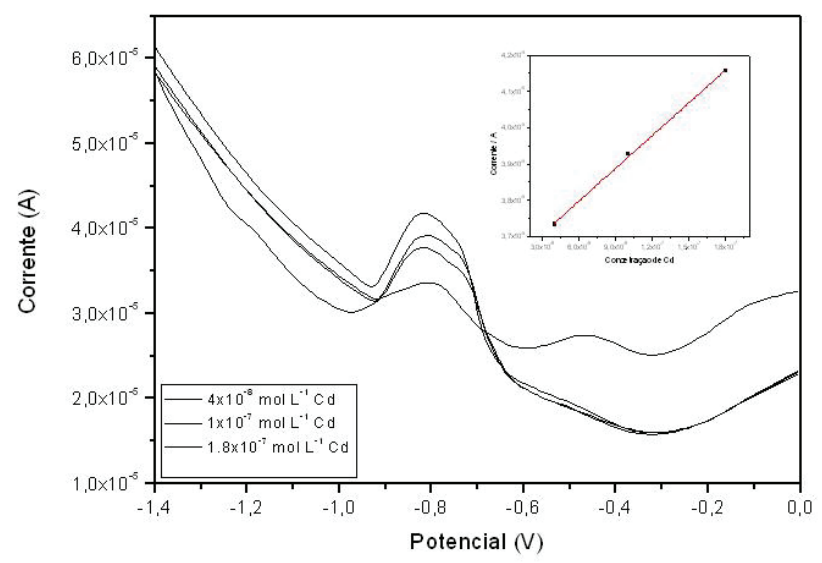

Fonte: Elaborada pelos autores

Conforme pode ser observado na figura 5 a resposta voltamétrica para $\mathrm{Cd}^{2+}$ foi boa em termos de sensibilidade, o que foi indicado pelo significativo aumento de corrente em função da adição de alíquotas sucessivas de $\mathrm{Cd}^{2+}$ na microemulsão estudada. Este gráfico foi obtido variando-se a concentração de $\mathrm{Cd}^{2+}$ entre $4 \times 10^{-8} \mathrm{M}$ a $1.8 \times 10^{-7} \mathrm{M}$, e a partir desses dados pôde-se construir uma curva analítica, que apresentou uma linearidade com coeficiente de correlação de 0.9977 e equação da reta: $A=3,618 \mathrm{e}^{-5}+30,03\left[\mathrm{Cd}^{2+}\right]$.

Com o objetivo de se predizer os resultados através de ferramentas computacionais, como redes neurais artificiais, que necessita de uma grande quantidade de pontos para fornecer resultados confiáveis, utilizou-se o método matemático de interpolação para gerar uma quantidade razoável de pontos a partir de resultados experimentais (correntes de $\mathrm{Cd}^{2+}$ e $\mathrm{Pb}^{2+}$ da figura 4), e então se gerou os gráficos mostrados abaixo na figura 6 e figura 7 para $\mathrm{Pb}^{2+} \mathrm{e} \mathrm{Cd}^{2+}$, respectivamente. 
Figura 6 - Curva analítica extraída da figura 4

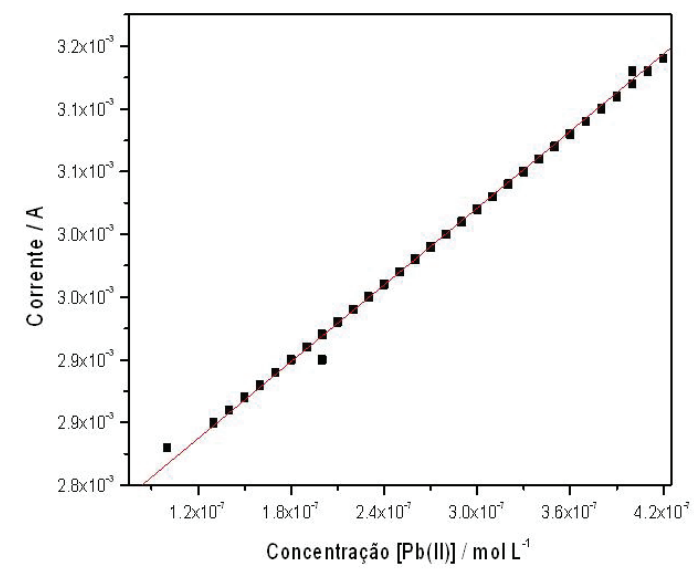

Fonte: Elaborada pelos autores

Figura 7 - Curva analítica extraída da figura 4

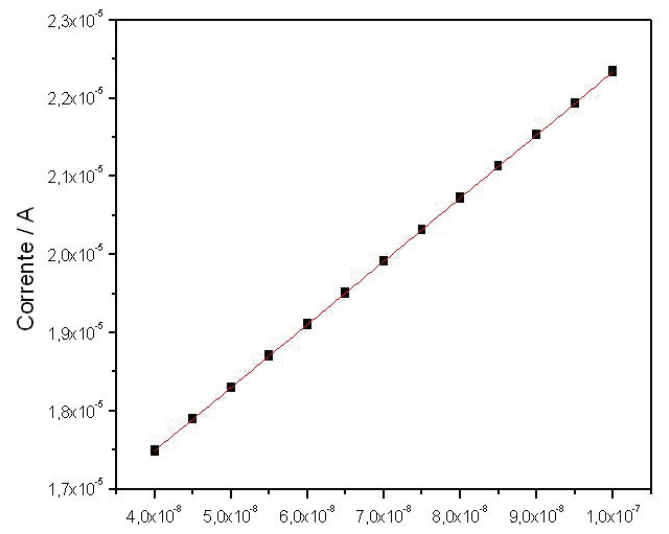

Fonte: Elaborada pelos autores

\section{Calibração multivariada}

No presente estudo usou-se o programa STATISTICA 10.0 na aplicação de redes neurais artificiais (RNA) para prever a concentração dos íons $\mathrm{Pb}^{2+}$ e $\mathrm{Cd}^{2+}$ em amostras microemulsionadas de gasolina.

Os resultados obtidos na curva de calibração foram processados em uma rede neural artificial do tipo MLP (Multi Layer Perceptron), sendo que esta foi treinada com o algoritmo de erro soma dos quadrados. Para ambos os metais, $40 \%$ dos pontos experimentais foram reservados para o treino da rede, 30\% para predição dos resultados e o restante para validação de seus respectivos resultados, as tabelas 4 e 5 mostram o coeficiente de correlação, em cada uma destas etapas.

Tabela 4 - Coeficiente de correlação no treino, predição e validação da rede neural Multi Layer Perceptron para o $\mathrm{Pb}^{2+}$.

\begin{tabular}{c|c|c|c}
\hline \multicolumn{4}{|c}{ Coeficiente de correlação } \\
\hline \multirow{3}{*}{$\begin{array}{c}\text { MLP } \\
1-4-1\end{array}$} & $\begin{array}{c}\text { Concentração } \\
\left(\text { mol L }^{-1)} \text { Tre- }\right. \\
\text { ino }\end{array}$ & $\begin{array}{c}\text { Concentra- } \\
\text { ção (mol L-1) } \\
\text { Predição }\end{array}$ & $\begin{array}{c}\text { Concentra- } \\
\text { ção (mol L } \\
\text { Validação }\end{array}$ \\
\cline { 2 - 4 } & 0,999 & 0,998 & 0,999 \\
\hline
\end{tabular}

Fonte: Elaborada pelos autores
Tabela 5 - Coeficiente de correlação no treino, predição e validação da rede neural Multi Layer Perceptron para $\mathrm{Cd}^{2+}$

\begin{tabular}{|c|c|c|c|}
\hline \multicolumn{4}{|c|}{ Coeficiente de correlação } \\
\hline \multirow{2}{*}{$\begin{array}{c}\text { MLP: } \\
1-2-1\end{array}$} & $\begin{array}{c}\text { Concentração } \\
(\text { mol L-1)Treino }\end{array}$ & $\begin{array}{c}\text { Concentra- } \\
\text { ção (mol L-1) } \\
\text { Predição }\end{array}$ & $\begin{array}{c}\text { Concentra- } \\
\text { cão }(\text { mol L-1 }) \\
\text { Validação }\end{array}$ \\
\cline { 2 - 4 } & 0,997 & 0,995 & 0,997 \\
\hline
\end{tabular}

Fonte: Elaborada pelos autores

Tabela 6 - Predição e validação da concentração de chumbo pela rede neural artificial

\begin{tabular}{|c|c|c|c|}
\hline \multicolumn{2}{|c|}{ Concentração $\left(\mu \mathrm{mol} \mathrm{L}^{-1}\right)$} & \multicolumn{2}{|c|}{ Concentração $\left(\mu \mathrm{mol} \mathrm{L}^{-1}\right)$} \\
\hline Predição & & Validação & \\
\hline $\begin{array}{l}\text { Valor real } \\
{[\mathrm{Pb}(\mathrm{II})]}\end{array}$ & $\begin{array}{l}\text { Valor encontrado } \\
\qquad[\mathrm{Pb}(\mathrm{II})]\end{array}$ & $\begin{array}{c}\text { Valor real } \\
{[\mathrm{Pb}(\mathrm{II})]}\end{array}$ & $\begin{array}{c}\text { Valor encontrado } \\
\qquad[\mathrm{Pb}(\mathrm{III})]\end{array}$ \\
\hline 0,200000 & 0,199346 & 0,130000 & 0,144715 \\
\hline 0,260000 & 0,260663 & 0,150000 & 0,153623 \\
\hline 0,280000 & 0,280235 & 0,160000 & 0,160603 \\
\hline 0,310000 & 0,309658 & 0,180000 & 0,178608 \\
\hline 0,330000 & 0,329520 & 0,220000 & 0,220385 \\
\hline 0,380000 & 0,380041 & 0,240000 & 0,240816 \\
\hline 0,390000 & 0,390218 & 0,250000 & 0,250795 \\
\hline 0,400000 & 0,400389 & 0,320000 & 0,319560 \\
\hline
\end{tabular}

Fonte: Elaborada pelos autores

Tabela 7 - Predição e validação de concentração de cádmio pela rede neural artificial

\begin{tabular}{|c|c|c|c|}
\hline \multicolumn{2}{|c|}{ Concentração $\left(\mu \mathrm{mol} \mathrm{L}^{-1}\right)$} & \multicolumn{2}{|c|}{ Concentração $\left(\mu \mathrm{mol} \mathrm{L}^{-1}\right)$} \\
\hline Predição & & Validação & \\
\hline $\begin{array}{l}\text { Valor real } \\
\text { [Cd (II)] }\end{array}$ & $\begin{array}{c}\text { Valor encontrado } \\
{[\mathrm{Cd}(\mathrm{II})]}\end{array}$ & $\begin{array}{l}\text { Valor real } \\
\text { [Cd (II)] }\end{array}$ & $\begin{array}{l}\text { Valor encontrado } \\
\qquad[\mathrm{Cd}(\mathrm{II})]\end{array}$ \\
\hline 0,010000 & 0,012376 & 0,030000 & 0,029307 \\
\hline 0,019000 & 0,018748 & 0,011000 & 0,012631 \\
\hline 0,021000 & 0,021004 & 0,013000 & 0,013474 \\
\hline 0,023000 & 0,023221 & 0,017000 & 0,016624 \\
\hline 0,026000 & 0,026212 & 0,020000 & 0,019871 \\
\hline 0,028000 & 0,027893 & 0,027000 & 0,027086 \\
\hline
\end{tabular}

Fonte: Elaborada pelos autores

Comparando os valores reais e os previstos conclui-se que tanto o modelo gerado pela rede neural para o chumbo (MPL 1-4-1) como para o cádmio (MPL 1-2-1) mostraram bons resultados, do ponto de vista de predição de concentrações na determinação simultânea dos analitos em estudo.

\section{CONSIDERAÇÕES FINAIS}

Neste trabalho apresentou-se um procedimento alternativo para a determinação simultânea dos ions $\mathrm{Pb}^{2+}$ e $\mathrm{Cd}^{2+}$ em amostras de gasolina microemulsionadas. Houve a necessidade de se descartar alguns dados experimentais de $\mathrm{Cd}^{2+}$, na determinação simultânea, devido a baixa reprodutibilidade na região deste ion, nas condições estudadas. A necessária complementação de dados, obtida por interpolação a partir de medidas individuais de $\mathrm{Cd}^{2+}$, nas mesmas condições e no intervalo de $2 \times 10^{-8} \mathrm{~mol} \mathrm{~L}^{-1}$ a $6 \times 10^{-8} \mathrm{~mol} \mathrm{~L}^{-1}$, mostrou-se eficiente porque os dados finais de predição 
foram muito satisfatórios. Isto foi comprovado pelo teste de recuperação em amostras reais. Os resultados otimizados por planejamento fatorial mostraram-se adequados e de grande sensibilidade analítica. O tipo de RNA usado (MLP) e os respectivos arranjos obtidos (MLP 1-4-1 para $\mathrm{Pb}^{2+}$, e MLP 1-2-1 para o $\mathrm{Cd}^{2+}$ ) foram capazes de estimar, muito satisfatoriamente, a concentração de cada analito na determinação simultânea em amostra real de gasolina $\left[\mathrm{Cd}^{2+}\left(\mathrm{R}^{2}=0,998\right)\right.$ e $\left[\mathrm{Pb}^{2+}\right]\left(\mathrm{R}^{2}=0,995\right)$. Desta forma, os valores simulados obtidos com a rede neural, corresponderam, estreitamente, aos resultados experimentais esperados. Do ponto de vista analítico, o procedimento foi aplicado com sucesso em amostras reais de gasolina, no nível de $10^{-9}$ e $10^{-8} \mathrm{~mol} \mathrm{~L}^{-1}$ para os íons $\mathrm{Pb}^{2+}$ e $\mathrm{Cd}^{2+}$, respectivamente.

\section{AGRADECIMENTOS}

UFMA (PIBIC, LAPQAP, LPQA), FAPEMA, UNB (LDCI e PGEA), FINEP (Projeto NANOPET), PETROBRAS (Projeto ELETROBIO), CAPES (Projetos PROCAD e PNPD)

\section{REFERÊNCIAS}

AGÊNCIA NACIONAL DO PETRÓLEO, GÁS NATURAL E BIOCOMBUSTÍVEIS. Resolução ANP no 57, de 20.10.2011. Diário Oficial da União, Brasília, DF, 21 out. 2011.

AUCÉLIO, R.Q.; CURTIUS, A.J. Evaluation of electrothermal atomic absorption spectrometry for trace determination of $\mathrm{Sb}$, As and Se in gasoline and kerosene using microemulsion sample introduction and two approaches for chemical modification. J. Anal. At. Spectrom., v. 17, p. 242-247, 2002.

AUCÉLIO, R.Q. et al. Electrothermal atomic absorption spectrometric method for the determination of vanadium in diesel and asphaltene prepared as detergentless microemulsions. Microchem. J., v. 78, p. 2126, 2004.

BARD, A. J.; FAULKNER, L. R. Electrochemical methods, fundamentals and applications. New York: John Wiley \& Sons, 2001.

BARROS NETO, B.; SCARMINIO, I. S.; BRUNS, R. E. Planejamento e otimização de experimentos. São Paulo: Ed. Unicamp, 1996.

Como fazer experimentos: pesquisa e desenvolvimento na ciência e na indústria. Campinas: Ed. da Unicamp, 2001.

BORSATO,D. et al. Kinetics of oxidation of biodiesel from soybean oil mixed with TBHQ: determination of storage time. Quím. Nova, v. 35, n. 4, p. 733-737. 2012. ISSN 0100-4042.

BUTTON, S. T. Metodologia para

planejamento experimental e análise de resultado. São Paulo: Universidade Estadual de Campinas. 2005. Apostila. Disponível em: <http://www.fem.unicamp.br/ sergio1/posgraduacao/IM317/apostila.pdf $>$. Acesso em: 31 dez. 2012.

CALADO, V.; MONTGOMERY, D. Planejamento de experimento usando o statistica. Rio de Janeiro: Ed. e- papers, 2003.

CARDOSO, C. E. et al. Voltammetric determination of copper and lead in gasoline using sample preparation as microemulsions. Analytical Sciences, v. 23, n. 9, p. 1065, 2007.

CARDOSO, W. S. et al. New $\mathrm{Pb}^{2+}$ carbon paste electrode based on organically modified silicate and its square wave anodic stripping voltammetric response for pretreated gasoline samples. Journal of the Brazilian Chemical Society, v. 21, p. 1733-1738, 2010.

CHAVES, E. S. et al. Determination of $\mathrm{Co}, \mathrm{Cu}, \mathrm{Fe}, \mathrm{Mn}, \mathrm{Ni}$ and $\mathrm{V}$ in diesel and biodiesel samples by ETV-ICP-MS. Journal of Environmental Monitoring, v. 10, p. 1211-1216, 2008.

CHRISTIAN, D. G. Analytical Chemistry. 4th ed., New York: John Wiley \& Sons, 1986.

COSTA, M. L. et al. Planejamento fatorial aplicado à digestão de feijão assistida por radiação microondas. Quim. Nova, v. 29, n. 29, n. 1, p. 149, 2006.

FERREIRA, J. M. et al. Estudo da remoção do cádmio em efluentes de indústrias Petroquímicas utilizando a Sacchromyces Cerevisiae. In: CONGRESSO BRASILEIRO DE P\&D EM PETRÓLEO E GÁS, 3., 2005, Salvador. Anais... Salvador: IBP, 2005.

HAYKIN, S. Neural networks: a comprehensive foundation. 2. ed. New Jersey: Prentice Hall, 1999.

LANDRIGAN, P. J. The worldwide problem of lead in petrol. Bulletin of World Health Organization, v. 80, n. 10, 2002.

MARQUES, A. L. B. et al. Controle de qualidade e métodos analíticos para derivados de petróleo e biocombustíveis. Eletroanálises de metais em combustíveis. Plural - Instituto Geia, p. 138-150, jun. 2012.

MARTINIANO, L. C. et al. Direct simultaneous determination of $\mathrm{Pb}$ (II) and $\mathrm{Cu}$ (II) in biodiesel by anodic stripping voltammetry at a mercury-film electrode using microemulsions. Fuel, v. 103, p. 1164-1167, 2013.

MONTGOMERY, D.C. Design and analysis of experiments. 3.ed. New York: John Wiley \& Sons, 1991.

SAINT' PIERRE, T. D. et al. Determination of $\mathrm{Cd}, \mathrm{Cu}, \mathrm{Fe}, \mathrm{Pb}$ and $\mathrm{Tl}$ in gasoline as emulsion by electrothermal vaporization inductively coupled plasma mass spectrometry with analyte addition and isotope dilution 
calibration techniques. Spectrochim. Acta Part B, v. 59, p. 551-558, 2004.

REYES, M. N. M.; CAMPOS, R. C. Graphite furnace atomic absorption spectrometric determination of $\mathrm{Ni}$ and $\mathrm{Pb}$ in diesel and gasoline samples stabilized as microemulsion using conventional and permanent modifiers. Spectrochimica Acta Part B: Atomic Spectroscopy, v. 60, p. 615-624, 2005.

RODRIGUES, M. I.; IEMMA, A. F. Planejamento de experimentos e otimização de processos. Campinas: Ed. Casa do Pão, 2005. 326p.

SANZ-MEDEL, A. et al. Organized surfactant assemblies in analytical atomic spectrometry. Spectrochim. Acta B, v. 54, p. 251-287, 1999.
SERRA, J. M. et al. Can artificial neral network help the experimental in catalysis? Catalysis Today, v. 18, p. 393-403, 2003.

TRINDADE, J. M. et al. Arsenic determination in gasoline by hydride generation atomic absorption spectroscopy combined with a factorial experimental design approach. Fuel, v. 85 , p. $2155-2161,2006$.

TRINDADE, J. M. et al. Anodic stripping voltammetry coupled with design of experiments for simultaneous determination of $\mathrm{Zn}^{+2}, \mathrm{Cu}^{+2}, \mathrm{~Pb}^{+2}$, and $\mathrm{Cd}^{+2}$ in gasoline. Fuel, v. 91, p. 26-32, 2012.

WYTHOFF, B. J. Backpropagation Neural Networks: a tutorial. Chemometrics and Intelligent Laboratory Systems, v. 18, p. 115 $-155,1993$. 